\title{
Do rodent species adopt to underground lifestyle by different ways?
}

\author{
O. Bondareva ${ }^{*}$, A. Kasianov ${ }^{2}$, N. Abramson ${ }^{1}$ \\ ${ }^{1}$ Zoological Institute RAS, Saint Petersburg, Russia \\ ${ }^{2}$ Vavilov Institute of General Genetics, Moscow, Russia \\ *e-mail:olga.v.bondareva@gmail.com
}

Key words: underground rodents, adaptations, gene ontology, underground lifestyle

Motivation and Aim: Up to now the majority of studies on the analysis of genetic diversity within species and populations was carried out using a small number of molecular markers. However, this approach does not account for the molecular basis of adaptive variation, it remains unclear how many genes involved in particular adaptation, what is the origin of genetic diversity responsible for formation of adaptation. Comparison of genomes of phylogenetically close taxa, but the contrast in adaptations and phylogenetically distant but with similar adaptive traits will recover convergence and parallelisms at the molecular level. This allows testing the hypothesis on the origin of mutations that lead to similar phenotype effects; reveal the velocity at which mutation in the DNA may cause a phenotypic effect.

Methods and Algorithms: We used annotated genomes of terrestical and underground rodents from the Ensembl genome browser (ensembl.org). Seven species with well assembled genomes were used: Cavia porcellus, Chinchilla lanigera, Rattus norvegicus, Mus musculus, Fukomys damarensis, Nannospalax galii, Heterocephalus glaber. Among listed organisms three are underground rodents. Ortholog genes were identified with protheinortho program and $\mathrm{dN} / \mathrm{dS}$ values were determined in PAML codeml program for each orthogroup. GO enrichment analysis was performed with Webgestalt software (http://www.webgestalt.org/option.php).

Results: First of all we tried to find out ortholog genes, for which directions of selection within analyzed groups is the same but differs between underground and terrestical species. Unfortunately, we could not identify such examples. Next we excluded from the analisis genomes of terrestical species and identified ortholog genes that have the same direction of selection in all underground species. These genes are enriched with several GO terms, connected with many essential processes: "RNA binding", "metabolic processes", "regulation" and "transcription activity" etc. The special interest represents groups of terms linked to mitochondria or oxidation processes, because they may be involved in the adaptation to hypoxia. This fact is good agreement that mitochondrial genes are under strong positive selection.

Conclusion: Obtained results demonstrate that adaptations to the underground lifestyle may be linked to changes in different essential processes.

Acknowledgements: Supported by the RFBR No. 18-04-00730. 